\title{
From loss to gain: role for SUN1 in laminopathies
}

\author{
Baohua Liu*, Dong-Yan Jin and Zhongjun Zhou*
}

\begin{abstract}
Mutations in $L M N A$ encoding lamins $A$ and $C$ are associated with at least 10 different degenerative disorders affecting diverse tissues, collectively called laminopathies. A recent study showed that mis-accumulation of SUN1 underlies the pathology of degenerative features in laminopathies, and concomitantly suggests a gain-of-function versus a loss-of-function model for the action of lamin A mutants.
\end{abstract}

The nuclear envelope (NE) is a double-layered membrane separating the cell's genetic material from the cytoplasm. The outer nuclear membrane (ONM) fuses with the inner nuclear membrane (INM) around the nuclear pore complex and extends to the endoplasmic reticulum (ER). Right underneath the INM is a layer of fine meshwork, namely nuclear lamina wherein various lamins and interacting proteins reside. A group of SUN (Sad1/UNC-84 homology) domain-containing proteins anchor to the INM and interact with different lamins, on the lamina, and nesprins, on the ONM, thus bridging nucleoskeleton and cytoskeleton. As one of the major components of the nuclear lamina (Figure 1), lamin A is firstly synthesized as prelamin A with an extra 18 amino acids at the C-terminus, which undergo transient isoprenylation, methylation, and these residues are finally removed by proteolytic cleavage [1]. To date, more than $237 L M N A$ mutations, leading to at least 163 protein variants, are known to be associated with at least 10 different degenerative disorders, collectively referred to as laminopathy. Some of these degenerative features are recapitulated in mice deficient for Lmna or harboring various Lmna mutations. Homozygous $L m n a^{L 530 P / L 530 P}$ mutation (Lmna $\Delta 9$ or Lamin A $\Delta$ Exon9) or loss of prelamin A-processing metalloproteinase Zmpste24 in mice phenocopies many of the progeroid features observed in Hutchinson-Gilford progeria syndrome (HGPS), one of the most severe forms of laminopathy that are predominantly caused by a 50 -amino-acid deletion in prelamin A (lamin $\mathrm{A} \triangle 50$ or progerin). Currently, the phenotypes in various mouse models and human diseases are thought

\footnotetext{
* Correspondence: ppliew@hku.hk; zhongjun@hku.hk

Department of Biochemistry, The University of Hong Kong, 3/F Laboratory Block, 21 Sassoon Road, Pokfulam, Hong Kong
}

to be attributable to either loss or gain of function of lamin A mutants.

A recent paper further highlights the gain-of-function versus loss-of-function model for the action of lamin A in laminopathies [2]. Chen et al. found that the level of Sun1 protein was up-regulated and mislocalized to Golgi in Lmna-/- and Lmna $\Delta 9$ cells. Although Golgi accumulation of SUN1 was not observed in HGPS cells, the levels of total and INM-localized SUN1 were significantly upregulated and well correlated with the misshapen nucleus, one of the hallmarks of HGPS fibroblasts and cells from mouse HGPS models [3,4]. SUN proteins are major components of the LINC (Linker of Nucleoskeleton and Cytoskeleton) complex, which links the nucleoskeleton and cytoskeleton, and mediates nucleus positioning. Further investigation showed that either increased INM-association of SUN1 in HGPS or its accumulation at Golgi in Lmna -/- and Lmna $\Delta 9$ cells was detrimental, as knocking down or genetically depleting SUN1 from the mutant cells largely rescued the misshapen nucleus and increased cell survival. Although all three mutant cells exhibited altered nuclear shape, they were virtually different. While loss of lamin A leads to partial loss of lamin B1 staining on NE, progerin only caused nuclear herniation in HGPS (see Figure 1). Consistent with this notion, Sun1 accumulated in the Golgi apparatus in Lmna-/- cells, but was properly localized on the NE in HGPS cells. In addition, while the majority of ectopic Sun1 can successfully anchor to NE, causing nucleus herniations, ectopic Golgi-orientated Sun1 led to partial loss of NE on the opposing site to the Golgi, mimicking Lmna null situation, in wild-type cells. Surprisingly, blocking SUN1 transportation from ER or NE towards Golgi by different small molecules restored the NE localization of both SUN1 and lamin B1. These data suggest that although lamin $\mathrm{A}$ is not required for the $\mathrm{NE}$
C Biomed Central (c) 2012 Liu et al.; licensee BioMed Central Ltd. This is an Open Access article distributed under the terms of the Creative Commons Attribution License (http://creativecommons.org/licenses/by/2.0), which permits unrestricted use, distribution, and reproduction in any medium, provided the original work is properly cited. 
A
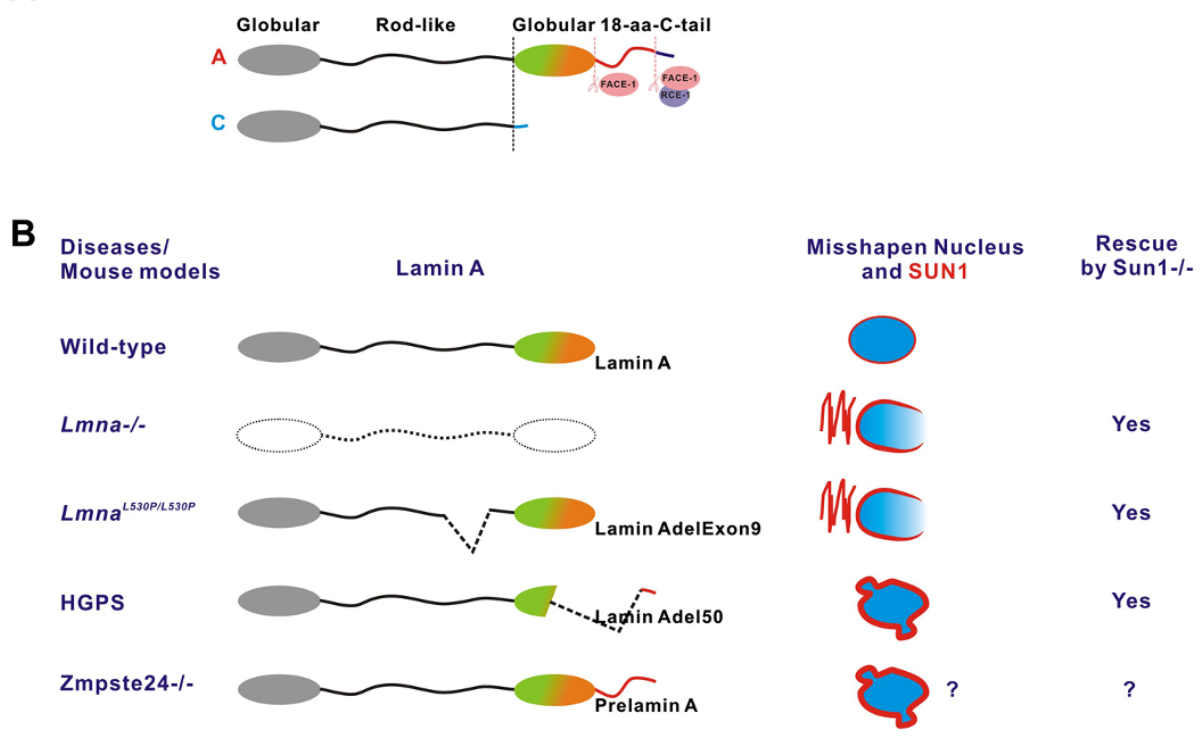

Figure 1 Summary of different lamin A mutants in human diseases and mouse models. A, Schematic diagram of lamin $A / C$ protein and post-translational processing of prelamin A. B, Status of lamin A and localization of SUN1 found in mouse models and human diseases.

positioning of SUN1, it may facilitate the retention of SUN1 on NE [5] and thus prevent SUN1 from accumulation at Golgi. Chen et al. and others also showed that farnesylated progerin/prelamin A increased the binding capacity to SUN1 [2,5]. In HGPS, it has been proposed that altered nuclear shape (herniation) is attributed to the accumulation of farnesylated progerin on the nuclear lamina as it can be rescued via treatment with farnesyl transferase inhibitors (FTIs); the rescue of the misshapen nucleus by reducing prelamin $\mathrm{A}$ or progerin from the nuclear lamina ameliorates senescence in HGPS cells [6,7] and progeroid features in progeria mouse models [7-9]. Remarkably, depleting SUN1 increased life spans in Lmna -/- and Lmna 9 mice and knocking down SUN1 in HGPS cells rescued heterochromatin loss and accelerated senescence.

The work by Chen et al. implicates a novel molecular mechanism for the pathogenesis of laminopathies; meanwhile it also raises many unanswered questions. Why does loss of lamin A delay the turnover of SUN1? What is the mechanism for the shuffling of SUN1 between Golgi and ER or NE? How does Golgi-accumulated SUN1 elicit degenerative phenotypes in tested mouse models? Why was Golgi-accumulated SUN1 not observed in HGPS cells? Answering these questions would help to better understand the molecular mechanism through which laminopathies are rescued by SUN1 depletion. The authors reasoned that the lacking Golgiaccumulated SUN1 in HGPS cells was attributable to a negative selection. Considering the loss-of-function versus gain-of-function model of lamin A proteins, there might be a second possibility. Given that the proper INM versus Golgi accumulation of SUN1 requires interaction with lamin A and increased binding capacity of SUN1 to farnesylated prelamin A, it is plausible to speculate that whereas loss of function of lamin A in Lmna-/- (loss of full-length lamin A), Lmna $\Delta 9$ (loss of exon 9 of lamin A) and HGPS (loss of 50 amino acids in one allele of $L M N A$ ) upregulates the level of SUN1 but loses the capacity to maintain its NE-anchorage; gain of function of progerin (lamin $\mathrm{A} \Delta 50$, gain of the farnesyl C-tail) attracts more SUN1 to the NE and leads to nucleus herniation. There could be a balance between loss and gain of function of progerin, and it would be worthwhile to examine if FTIs could remove SUN1 from the $\mathrm{NE}$ and whether this underlies the rescuing effect of FTIs in HGPS. To further test this hypothesis, it would be of interest to determine the level of Sun1 in Zmpste24-/- mice, as well as cells ectopically expressing progerin. If this is true, one would expect no abnormal Golgi-accumulation of SUN1 but increased NEassociated Sun1 in Zmpste24 null mice due to the presence of prelamin A protein with a farnesyl C-tail. In fact, it has been recently shown that, while both prelamin $\mathrm{A}$ and progerin exhibited increased binding capacity to histone $\mathrm{H} 3$ peptide in comparison with lamin A, probably attributable to their gain of function, they showed differences in binding to H3K27 peptide [10]. On the other hand, prelamin A and lamin A had similar binding capacity to H3K27 peptide, whereas progerin had a reduced binding capacity, possibly due to a loss of function. It was proposed that these functional variations are likely 
attributable to structural differences, i.e. whereas both prelamin A and progerin contain an extra farnesylated and methylated C-terminal tail, the 50-amino-acids deletion differentiates progerin from prelamin A.

\section{Competing interests}

The authors declare that they have no competing interests.

\section{Acknowledgment}

Research work by B.L. was supported in part by Seed Funding for Basic Research from the University of Hong Kong. Research work in Z.Z. lab was supported by Progeria Research Foundation, Research Grants Council of Hong Kong (HKU7655/06M, CRF/HKU3/07C) and Ministry of Science and Technology of China (2007CB507400).

\section{Authors' contributions}

BL, DYJ and ZZ drafted the manuscript. All authors read and approved the final manuscript.

Received: 1 June 2012 Accepted: 6 June 2012

Published: 18 June 2012

\section{References}

1. Rusinol AE, Sinensky MS: Farnesylated lamins, progeroid syndromes and farnesyl transferase inhibitors. J Cell Sci 2006, 119:3265-3272.

2. Chen CY, Chi YH, Mutalif RA, Starost MF, Myers TG, et al: Accumulation of the inner nuclear envelope protein sun 1 is pathogenic in progeric and dystrophic laminopathies. Cell 2012, 149:565-577.

3. Liu B, Wang J, Chan KM, Tjia WM, Deng W, et al: Genomic instability in laminopathy-based premature aging. Nat Med 2005, 11:780-785.

4. Pendas AM, Zhou Z, Cadinanos J, Freije JM, Wang J, et al: Defective prelamin A processing and muscular and adipocyte alterations in Zmpste24 metalloproteinase-deficient mice. Nat Genet 2002, 31:94-99.

5. Crisp M, Liu Q, Roux K, Rattner JB, Shanahan C, et al: Coupling of the nucleus and cytoplasm: role of the LINC complex. J Cell Biol 2006, 172:41-53.

6. Toth Jl, Yang SH, Qiao X, Beigneux AP, Gelb MH, et al: Blocking protein farnesyltransferase improves nuclear shape in fibroblasts from humans with progeroid syndromes. Proc Natl Acad Sci U S A 2005, 102:12873-12878.

7. Capell BC, Erdos MR, Madigan JP, Fiordalisi JJ, Varga R, et al: Inhibiting farnesylation of progerin prevents the characteristic nuclear blebbing of Hutchinson-Gilford progeria syndrome. Proc Natl Acad Sci U S A 2005, 102:12879-12884.

8. Fong LG, Frost D, Meta M, Qiao X, Yang SH, et al: A protein farnesyltransferase inhibitor ameliorates disease in a mouse model of progeria. Science 2006, 311:1621-1623.

9. Varela I, Pereira S, Ugalde AP, Navarro CL, Suarez MF, et al: Combined treatment with statins and aminobisphosphonates extends longevity in a mouse model of human premature aging. Nat Med 2008, 14:767-772.

10. Bruston F, Delbarre E, Ostlund C, Worman HJ, Buendia B, et al: Loss of a DNA binding site within the tail of prelamin A contributes to altered heterochromatin anchorage by progerin. FEBS Lett 2010, 584:2999-3004.

\section{Submit your next manuscript to BioMed Central and take full advantage of:}

- Convenient online submission

- Thorough peer review

- No space constraints or color figure charges

- Immediate publication on acceptance

- Inclusion in PubMed, CAS, Scopus and Google Scholar

- Research which is freely available for redistribution 